\title{
Virtual mask fitting in pediatric patients during COVID-19: A case series
}

Tuyen Tran HBSc, RRT ${ }^{*}$, Mika Nonoyama RRT, PhD ${ }^{2,3,4}$, Nisha Cithiravel HBSc, RRT ${ }^{1}$, Faiza Syed HBSc, RRT ${ }^{1}$, Joanna Janevski RN, NP ${ }^{5}$, Jackie Chiang MA MD ${ }^{5}$, Reshma Amin MD MSc, FRCP 5

\begin{abstract}
T. Tran, M. Nonoyama, N. Cithiravel, F. Syed, J. Janevski, J. Chiang, R. Amin Virtual mask fitting in pediatric patients during COVID-19: A case series Can J Respir Ther 2021;57:93-98. doi: 10.29390/cjrt-2021-023.

Introduction: The COVID-19 pandemic has been an unprecedented threat to our health care system. Clinicians had to pivot and develop creative and timely "virtual" solutions to provide clinical care. Our aim was to develop a standardized approach to virtual "mask fitting" for children who are either being initiated or are already on existing long-term ventilation (LTV) at a pediatric hospital.

Case and outcomes: We present three cases involving the care of children who required mask fitting for noninvasive ventilation (NIV). LTV team consultations were delivered via videoconference or phone. With the guidance of the respiratory therapist (RT), the family caregiver (FC) took measurements on their child using a standardized clinical approach (developed by the LTV RTs). Based on the measurements, an appropriate mask was selected. Successful mask fit was based on patient/FC reports, as well as objective leak data obtained from the NIV download data.

Discussion: Virtual clinics used for managing patients in our LTV program were feasible and efficient resulting in improved workflow for the RTs and convenience for patients and FCs. Patients and FCs had significantly less pressure to attend in-person clinics and expressed high satisfaction in terms of their experience and importantly, meeting respiratory care needs. Within the context of COVID-19, remote patient education and intervention can be delivered effectively, while reducing the risk of exposure from in-person visits to hospital.

Conclusion: A virtual/telemedicine program to manage pediatric patients requiring mask fitting for LTV was a feasible option during COVID-19.
\end{abstract}

Key Words: long-term ventilation; NIV; facial; nasal; full face; measurement, respiratory therapist; template

\section{INTRODUCTION}

The global pandemic from the severe acute respiratory syndrome coronavirus 2 (SARS-Co-V-2) has significantly impacted the delivery of ambulatory care services across the health system [1]. In March 2020, due to the increasing number of COVID-19 cases, the Ontario Ministry of Health and Long-Term Care requested that hospitals ramp down elective surgeries and other nonemergent clinical activity.

Virtual care platforms include telephone or video conferencing and allow health care patient-clinician interactions to take place outside of in-person hospital settings [2]. Virtual appointments during pandemic times, among physicians and adult patients, have lessened the transmission of the virus, thus protecting both parties from infection [3]. In contrast, the role of remote consultations for pediatric patients remains unclear, as the infection rates among children are lower compared with adults. Despite children being susceptible, they develop milder COVID19 related symptoms; severe complications and mortality are rare in children, even those with underlying medical conditions [4].

In response to the pandemic, the long-term ventilation (LTV) clinic at the Hospital for Sick Children (SickKids) transitioned all in-person appointments to virtual. Prior to or during the virtual clinic appointment, family caregivers (FCs) either emailed or mailed in their child's ventilator or noninvasive ventilation (NIV) device data for analysis by the respiratory therapists (RTs). SickKids RTs developed a standardized clinical approach to virtual "mask fitting" for children who were being initiated or already on NIV for LTV. This was a necessity as most vendors that sold respiratory equipment were no longer providing in-person mask fitting sessions at the beginning of the pandemic.

\section{MASK FIT PROCEDURES}

With guidance from RTs, FCs measured their child's face with a measuring tape, using a standardized clinical approach to mask fit. FCs provided information about their child's sleeping position, presence of snoring, frequency of movement during sleep, availability of nursing care, and skin integrity. The measurements and responses to the questions informed the choice of the interface.

For nasal mask (NM) sizing, two fiducial facial measurements were taken, including height of the nose from the bridge to the bottom of the nose and width of the nose from side to side (Figure 1). Full-face mask (FFM) measurements included height of the nose from under the lip to the bridge of nose and width of the mouth at the widest point (Figure 2).

RTs used proprietary fitting templates available in the mask packages, or found online, to help determine mask sizing. The width or height of

\footnotetext{
${ }^{1}$ Department of Respiratory Therapy, The Hospital for Sick Children, Toronto, Canada;

${ }^{2}$ Department of Respiratory Therapy $\mathcal{E}$ Child Health Evaluative Sciences, The Hospital for Sick Children, Toronto, Canada;

${ }^{3}$ Faculty of Health Sciences, Ontario Tech University, Oshawa, Canada;

${ }^{4}$ Rehabilitation Sciences Institute $\mathcal{E}$ Department of Physical Therapy, University of Toronto, Toronto, Canada;

${ }^{5}$ Division of Respiratory Medicine, The Hospital for Sick Children, Toronto, Canada;

${ }^{6}$ University of Toronto, Toronto, Canada

Correspondence: Tuyen Tran, Department of Respiratory Therapy, The Hospital for Sick Children, 555 University Avenue, Toronto, Ontario, Canada, M5G

1X8. Tel: 416-813-6274,E-mail: tuyen.tran@sickkids.ca
}

Published online at https://www.cjrt.ca on 23 July 2021 


\section{FIGURE 1}

Fiducial facial landmarks for nasal mask fitting. Height of the nose from the bridge to the bottom of the nose (blue line) and width of the nose from side to side (red line).

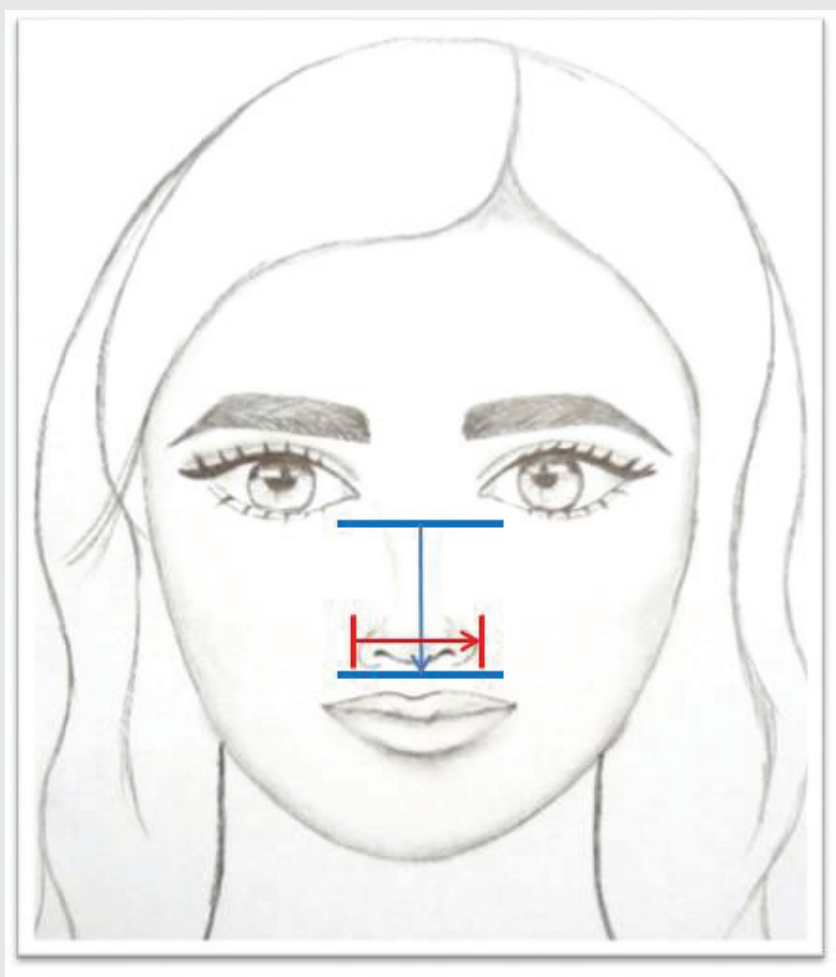

the size guides on the templates (Figure 3) were manually measured. For masks that did not come with a fitting template, two measurements were required; the height of the mask from the top to the bottom opening and the widest part of the inner opening (Figure 4). A "master" mask table was then developed and included the measurements of the different sizes and brands of masks commonly used in our LTV practice. Figure 5 provides a flow diagram of the overall virtual mask fitting process.

Research Ethics Board approval for this project was not required by the institution. SickKids case report consent form was obtained for each participant of the case study.

\section{CASE PRESENTATIONS}

The patients in this report were selected because they demonstrated a diversity of cases including (i) new initiation of NIV, (ii) spontaneous/ timed (ST) mode with large leak, and (iii) volume assured pressure support mode with large leak. These three different and complex patients highlighted different situations, different therapies, and challenges RTs experience in the LTV clinic. In total, we completed virtual mask fittings on 20 patients $(n=9$ had virtual mask fit process + virtual appointment; $n=11$ had virtual mask fit process applied with the in-person appointment). After the virtual mask fit appointments, patients are followed up via telephone calls or emails in approximately 2 weeks (to check on mask fit) and 1 month (for NIV downloads).

\section{Case 1}

\section{Clinical presentation}

Case 1 was an 8-year-old female diagnosed with Congenital Myopathy and scoliosis. A prior sleep study showed an elevated carbon dioxide level (45-50 $\mathrm{mmHg})$ via transcutaneous carbon dioxide $\left(\mathrm{TCCO}_{2}\right)$ monitoring with normal oxygen saturation. She also had ongoing feeding intolerance, poor weight gain, decreased energy, lethargy, and reduced self-ambulation. She presented with a significant decline in motor strength compared to the past year, which affected her mobility. She had a repeat sleep study a year and a half later, showing moderate obstructive sleep apnea, significant central sleep apnea, mild nocturnal hypoventilation by $\mathrm{TcCO}_{2}$ criteria, and prolonged oxygen desaturation with poor recovery to baseline. Based on the results of this polysomnogram, the decision was made to initiate NIV, requiring the patient to go to SickKids for a mask fit.

However, the $\mathrm{FC}$ was hesitant to participate in an in-person mask fitting due to the length of the commute to the hospital, her daughter's high anxiety issues, and fears regarding potential exposure to COVID-19. Therefore, a virtual mask fitting appointment was scheduled with the SickKids RT.

\section{Intervention}

The virtual visit was completed. Facial measurements for both nasal mask (nose height $=6.0 \mathrm{~cm}$, nose width $=4.5 \mathrm{~cm}$ ) and FFM (nose height $=9.0 \mathrm{~cm}$, mouth width $=5.0 \mathrm{~cm}$ ) were completed. The FC chose one large NM with a chin strap (she slept with her mouth open) and one medium FFM to try. During this virtual visit, mask fitting was completed in addition to extensive NIV education. The masks were then delivered from SickKids to the peripheral community hospital where she had an appointment 1 week later for NIV initiation and acclimation. The community RT reported that the FC preferred the FFM due to comfort and fit for her daughter and her daughter refused to wear the chinstrap.

\section{Patient and family perspective}

This virtual mask fitting session had several benefits. The SickKids RT, together with the community hospital RT, were able to expedite the mask fitting, allowing the patient to be initiated on NIV. The patient and FC reported satisfaction with the virtual visit and a reduction in stress and anxiety because they did not have to commute to multiple hospitals.

\section{Case 2}

\section{Clinical presentation}

Case 2 was a 16 -year-old male with a medical history including cerebral palsy, chronic lung disease of prematurity, and neuromuscular 


\section{FIGURE 2}

Fiducial facial landmarks for full face mask fitting. Height of the nose from under the lip to the bridge of nose (blue line) and width of the mouth at the widest point (red line).

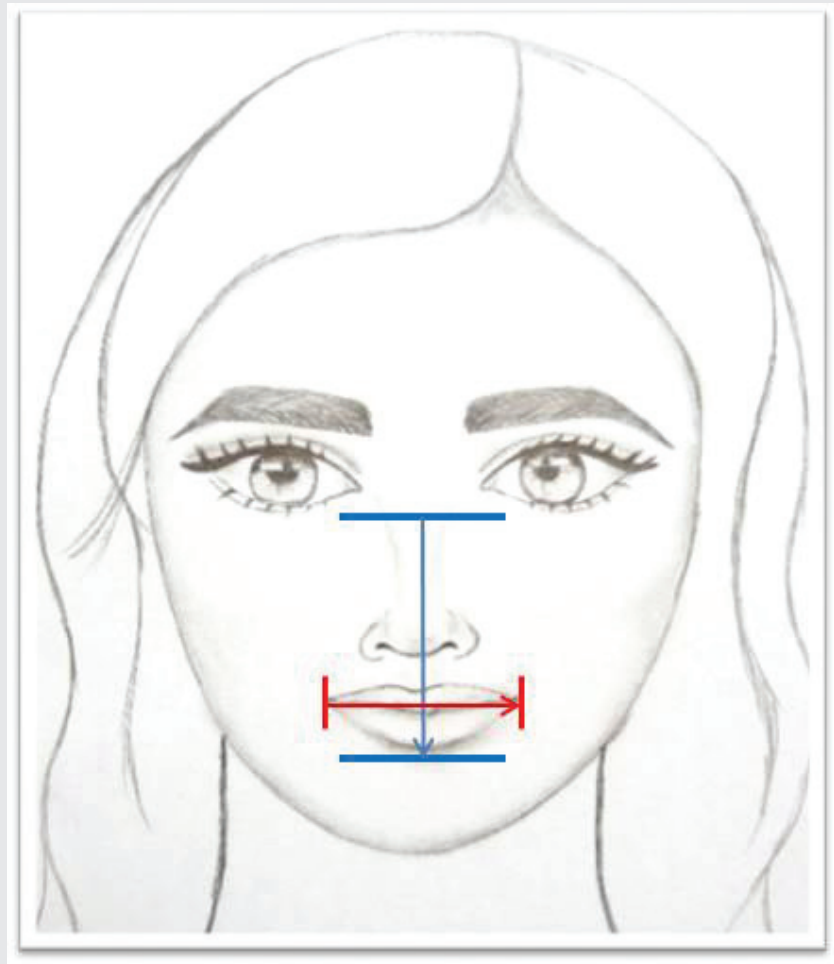

\section{FIGURE 3}

Examples of fitting templates/guides and manual measurements made by the RT (red lines) for the master mask table (Resmed, San Diego, CA, United States). Retrieved from: https:www.resmed.com/us/dam/documents/products/Mask/airfit-n20/ fitting-guide/airfit-n20_fitting_template_row_eng.pdf and https://document.resmed.com/en-us/documents/products/mask/airfit-f20/ fitting-guide/airfit-f20_fitting-temp-web-inch_amer_eng.pdf

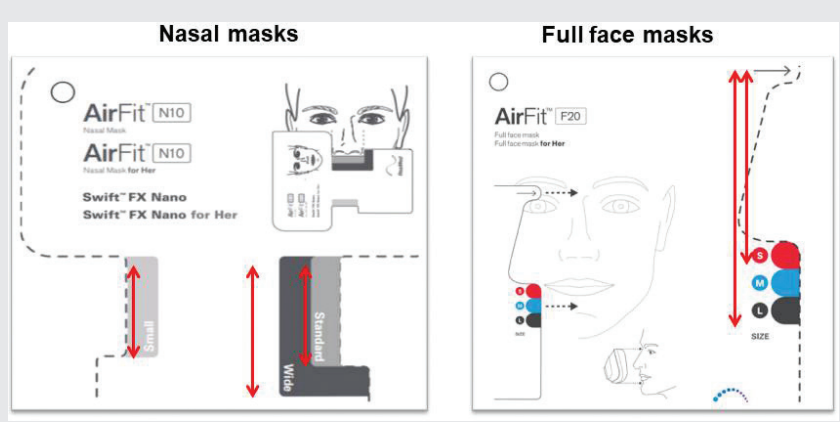

scoliosis with lifelong use of NIV. He was using ST mode, with inspiratory positive airway pressure $18 \mathrm{cmH}_{2} \mathrm{O}$, expiratory positive airway pressure (EPAP) $9 \mathrm{cmH}_{2} \mathrm{O}$ and a back-up rate (BUR) 18 breaths per minute (bpm). The NIV data download showed good adherence (approximately $9 \mathrm{~h}$ per night), but there were significant leaks, and the FC reported High Leak alarms overnight. The median unintentional mask leak was $43 \mathrm{~L}$ per minute $(\mathrm{Lpm})$ and the 95 th percentile was $63 \mathrm{Lpm}$ (above the manufacturer's threshold for acceptable unintentional mask leak). The patient was using a small FFM that fit under his nose and around the mouth, purchased less than 6 months prior.

\section{Intervention}

The interface issue was discussed during a scheduled virtual clinic appointment with the LTV team. First, the causes of the mask leaks were discussed with the patient. These included improper fit and size, use of older masks, soiled masks, sleep position, high air pressure, and/or breathing through the mouth. Second, potential risks and challenges associated with mask leak 


\section{FIGURE 4}

Example of a mask without a template/guide and manual measurements. Height of the mask was measured from the top of the mask to the bottom opening of the mask (blue line). The width of the mask was widest part of the inner opening from one side of the mask to the other (red line).

were discussed with both the patient and FC. These included dryness, irritation of the eyes due to the air blowing, sleep disturbances/fragmentation caused by mechanical whistling or hissing noises, and inadequate ventilation and stomach bloating that can cause discomfort in the morning.

Upon assessment of the patient's mask interface, there were leaks noted above and below the mask. A NM with a chin strap was not an option because this was tried previously without success. The patient's fiducial landmarks using the FFM procedure were nose height $=10.0 \mathrm{~cm}$, mouth width $=8.0 \mathrm{~cm}$. Obtaining accurate measurements was challenging as the patient was smiling frequently. Due to concerns about the accuracy of the measurements (over-estimation), both a large and medium size FFMs were provided that fit above the nose and below the lips. The masks were mailed to the family. A couple of weeks later, the RT called and asked the FC to fit the two masks on her child. The FC noted the large mask had a lot of air leaking around the nose, and the leak reading on the NIV was high. The medium mask with head gear adjustments resulted in improved leak reading. The decision was to use the medium mask. A month later a repeat NIV download showed the median leak was reduced to $27 \mathrm{Lpm}$ compared to $43 \mathrm{Lpm}$ previously with the medium size mask.

\section{Patient and family perspective}

The FC stated that she liked the process of the virtual mask fitting because it was convenient and very "solution oriented." This was especially true given the patient was nonambulatory, and it took more effort and time to go to vendors to buy a mask. Both masks were mailed to the family to help with finances, and to prevent delays in optimizing the NIV therapy. Optimizing therapy with a proper mask seal allowed the patient to get restful sleep, increased energy levels, and improved concentration.

Case 3

\section{Clinical presentation}

Case 3 was a 16-year-old male diagnosed with Spinal Muscular Atrophy Type 2 (SMA2). Children with SMA2 have generalized muscle weakness compromising their respiratory muscles (inspiratory and expiratory) and mobility. They have weak respiratory muscles, making breathing more difficult. Short and shallow breathing during sleep can also result in poor oxygen and carbon dioxide exchange. Children with SMA2 are typically placed on NIV nocturnally to help build their respiratory strength by allowing them to rest, giving them more energy throughout the day. He used a volume assured pressure support mode with his NIV with a target alveolar ventilation $5 \mathrm{Lpm}$, BUR $15 \mathrm{bpm}$, EPAP $6 \mathrm{cmH}_{2} \mathrm{O}$, pressure support (PS) minimum $6 \mathrm{cmH}_{2} \mathrm{O}$ and $\mathrm{PS}$ maximum $16 \mathrm{cmH}_{2} \mathrm{O}$. This patient had a long-standing leak issue with a NM and chin strap, but was not keen on trying a FFM. Large mask leaks in this particular mode can cause swings in pressure support and patient discomfort by increasing air in the stomach, resulting in bloating, abdominal pain, nausea, vomiting, and risk of aspiration. Fixing any mask leak can drastically improve the efficacy of ventilation. During his last LTV clinic visit he had an unintentional leak of $12 \mathrm{Lpm}$ and 95 th percentile of 33 $\mathrm{Lpm}$. He was advised to replace the chin strap since he had been using it for over 6 months. A repeat download was completed in preparation for the virtual clinic appointment, and showed an improvement in the unintentional leak with a median of $7.4 \mathrm{Lpm}$ and 95 th percentile of 23.7 Lpm.

\section{Intervention}

The virtual visit was completed via phone-call. This time, the patient was willing to try a FFM. The measurements were nose height $=8.1 \mathrm{~cm}$, mouth width $=5.0 \mathrm{~cm}$. Based on the virtual assesssment and measurements, the RT mailed a small FFM to the patient. A scheduled follow up was completed 1 week later. Both the patient and FC stated the mask was a good fit but there was still a small leak around the nose. He was advised to use a nasal gel pad, which he had used for a previous mask. A follow up NIV data download 1 month later showed a significant improvement in the unintentional mask leak with a median of $1.7 \mathrm{Lpm}$ and 95th percentile of $12.5 \mathrm{Lpm}$. 


\section{FIGURE 5}

Flow diagram of virtual mask fitting process in the long-term ventilation clinic during a virtual appointment.

Flow Diagram of Virtual Mask Fitting

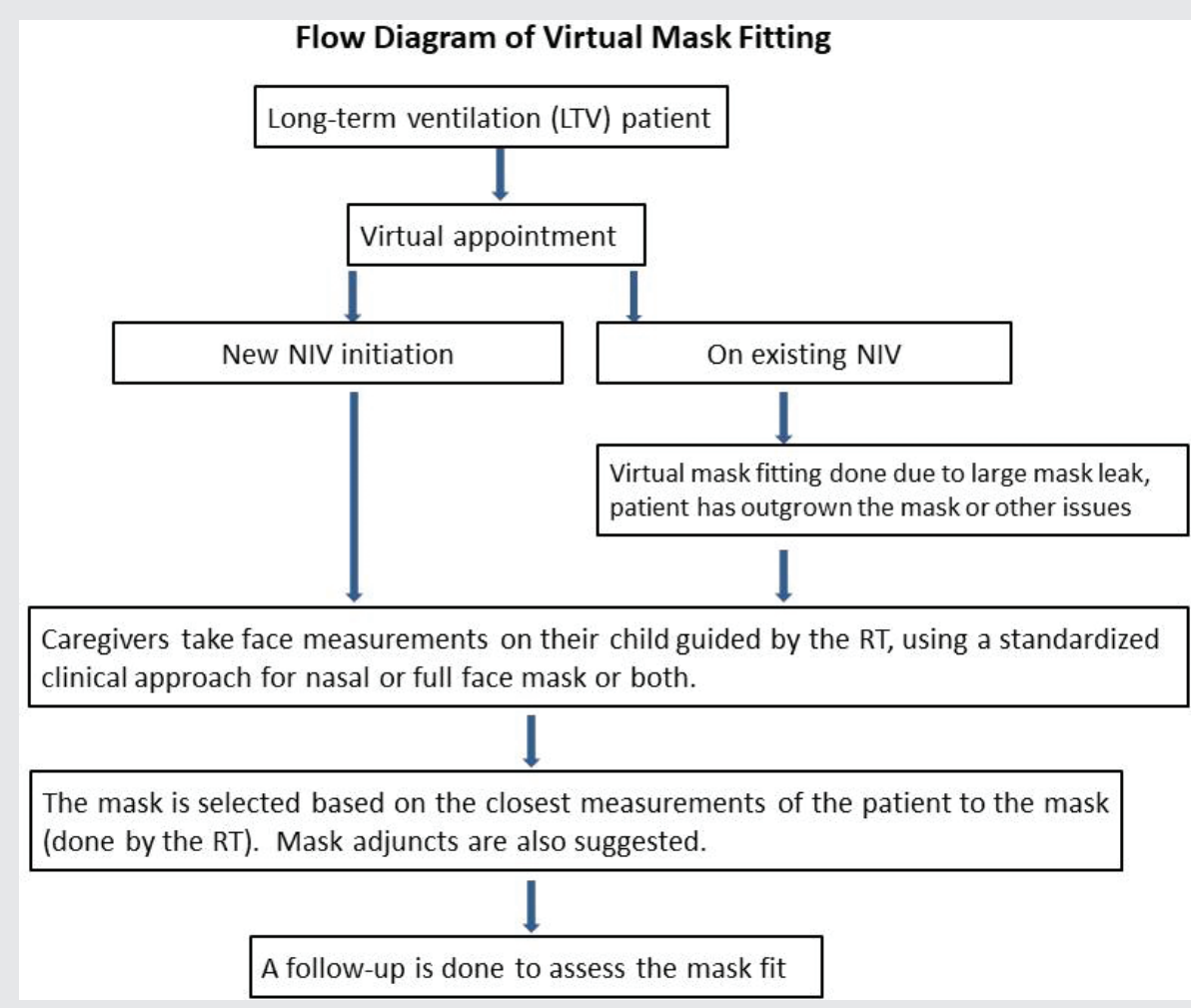

\section{Patient and family perspective}

Despite the patient not liking the feel of the FFM, the FC noticed a big difference. There were less High Leak alarms overnight, which decreased the number of times the FC had to get up to reposition her son and adjust the mask. This in turn allowed the patient to sleep better, to have fewer headaches, and better overall concentration during the day.

\section{DISCUSSION}

In this case series, we reported the clinical experiences of a virtual patient care environment within a pediatric respiratory care setting. Prior to COVID-19, multiple masks were applied on a trial and error basis to achieve mask fit. Our virtual LTV clinic successfully used the facial and mask measurement process to determine the right mask fit for our patients. In this case series, one patient was initiated on NIV while the second and third patient had reduced mask leak due to the new fitted masks. In addition, patients and FCs were able to remain safely in their homes and were satisfied with their patient care experience. Because the masks were fitted virtually, fewer masks were discarded, reducing supply costs. Future studies could examine if this process reduces overall costs for both the families and the health care system. It is anticipated that even in the post-COVID era, virtual clinics like ours will continue to grow in practice. Video consultation appointments during the pandemic increased by more than $12,000(72 \%)$ at a large medical centre in California, USA [5]. This was similar to the experience in our LTV clinic between March 2020 to September 2020, where approximately $89 \%$ of appointments were completed virtually. The usefulness of telemedicine in the management of asthma [6], chronic obstructive pulmonary disease $[7,8]$, and sleep apnea [9] have been demonstrated in previous reports. These studies compared virtual processes with in-person care. Telemedicine care involved case management, consultation, education, monitoring, and patient reminders $[6,8]$ as well as pulmonary rehabilitation [7]. Telemedicine management was feasible $[6,7]$ and resulted in improvements in asthma control and outcomes [6] and enhanced quality of life [6, 7]. These studies demonstrated that telemedicine approaches are equivalent to face-to-face care in certain circumstances but were conducted in adult populations and during nonpandemic times.

There are some notable limitations to our findings. This case series report included a small cross-section of the patient population at a tertiary care children's hospital and may not be generalizable to other clinical practices. Additionally, the long-term efficacy of the virtual mask fittings is unknown because follow up consultations were not completed over an extended time interval. This report is meant to inform a future prospective study including formal patient and family satisfaction questionnaires as well as clinical outcome measures.

\section{CONCLUSION}

This case series demonstrates that the LTV virtual care mask fitting process was highly collaborative and helped guide and educate pediatric patients and FCs on mask fitting procedures. Patients and FCs were satisfied because of the reduced travel time, and decreased anxieties associated with potential COVID-19 exposure. RT workload and confidence in the mask fit process improved, and there may have been cost savings from decreased use of mask supplies in these three cases. Larger prospective studies are required to determine the impact of virtual care clinics in the LTV pediatric population on patient outcomes (including quality of life), health utilization, and costs.

\section{AUTHOR DISCLOSURES}

\section{Contributors}

TT conceptualized the concept and wrote the initial draft of the manuscript. TT, NS, and FS collected the data. MN, RA, JC, NS, FA, JP critically reviewed all of the manuscript drafts and provided scientific feedback. All authors approved the final version of the manuscript. 


\section{Tuyen Tran et al.}

\section{Funding}

This study did not receive any specific grant from funding agencies in the public, commercial or not-for-profit sectors.

\section{Competing Interests}

All authors have completed the ICMJE uniform disclosure form at www. icmje.org/coi_disclosure.pdf and declare: no financial relationships with any organizations that might have an interest in the submitted work in the previous 3 years; no other relationships or activities that could appear to have influenced the submitted work.

\section{Ethical Approval}

REB approval for this project was not required by the institution. SickKids case report consent form was obtained for each of the case study.

\section{REFERENCES}

1. Barrett K, Khan YA, Mac S, Ximenes R, Naimark DMJ, Sander B. Estimation of COVID-19-induced depletion of hospital resources in Ontario, Canada. Can Med Assoc J 2020;192(24):E640-6. doi: 10.1503/ cmaj.200715

2. Ajami H, Mcheick $H$, Mustapha K. A pervasive healthcare system for COPD patients. Diagnostics 2019;9(4):135. doi: 10.3390/ diagnostics 9040135
3. Anthony B, Jnr. Use of telemedicine and virtual care for remote treatment in response to COVID-19 pandemic. J Med Syst 2020;44(7):132. doi: 10.1007/s10916-020-01596-5

4. Al-Hajjar S, McIntosh K. Pediatric COVID-19: an update on the expanding pandemic. Int J Pediatr Adolesc Med 2020;7(2):61-3. doi: 10.1016/j. ijpam.2020.05.001

5. Lonergan PE, Washington III SL, Branagan L, et al. Rapid utilization of telehealth in a comprehensive cancer center as a response to COVID-19: cross-sectional analysis. J Med Internet Res 2020;22(7):e19322. doi: $10.2196 / 19322$

6. Chongmelaxme B, Lee S, Dhippayom T, Saokaew S, Chaiyakunapruk N, Dilokthornsakul P. The effects of telemedicine on asthma control and patients' quality of life in adults: a systematic review and meta-analysis. J Allergy Clin Immunol Pract 2019;7(1):199-216.e11. doi: 10.1016/j. jaip.2018.07.015

7. Jiang Y, Liu F, Guo J, et al. Evaluating an intervention program using WeChat for patients with chronic obstructive pulmonary disease: randomized controlled trial. J Med Internet Res 2020;22(4):e17089. doi: 10.2196/17089

8. Trosini-Désert V, Lafoeste H, Regard L, et al. A telemedicine intervention to ensure the correct usage of inhaler devices. Telemed e-Health 2020;26(11):1336-44.

9. Bruyneel M. Telemedicine in the diagnosis and treatment of sleep

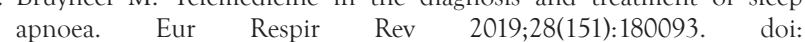
10.1183/16000617.0093-2018 